\title{
From Order to Disorder of Alkanethiol SAMs on Complex Au (211), (221) and (311) Surfaces: Impact of the Substrate
}

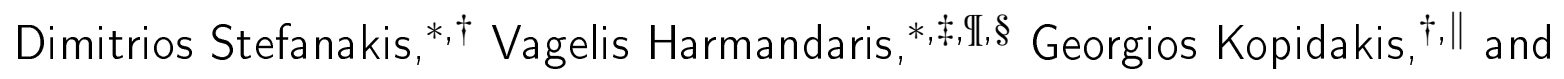
loannis Remediakis ${ }^{\dagger}, \|$

$\dagger$ Department of Materials Science \& Technology - University of Crete, Vassilika Voutes, 70013 Heraklion, GREECE

$¥$ Department of Mathematics \& Applied Mathematics - University of Crete, Vassilika Voutes, 700 13 Heraklion, GREECE

IIInstitute of Applied \& Computational Mathematics, Foundation for Research and Technology-Hellas, 71110 Heraklion, GREECE

§omputation-Based Science and Technology Research Center, The Cyprus Institute, Nicosia 2121, Cyprus

||Institute of Electronic Structure and Laser, Foundation for Research and Technology-Hellas, 711 10 Heraklion, GREECE

E-mail: dimstef@materials.uoc.gr; harman@uoc.gr 


\section{Supporting material}

\section{Fitting Data for the calculated potential}

The calculation process of the fitting data is demonstrated in Table S1. The table is divided in four sections that represent each one of the studied surfaces, as indicated. Each section is divided in three columns:

1. Starting from the third column of each section, the difference between the absolute value and the lowest absolute value of calculated potential in $\mathrm{kJ} / \mathrm{mol}$ is demonstrated. Thus, a zero value indicates the configuration of the lowest potential for each system.

2. The first column of each section indicates the dihedral angle of Au-S-C $\mathrm{C}^{(1)}-\mathrm{C}^{(2)}$ starting from our initial configuration ( $0 \mathrm{deg}$ ). This was retrieved from data reported by Barmparis et al.[citation 18 in the paper manuscript] as the configuration of lowest adsorption energy for each surface. The fact that the initial angle generally does not correspond to the lowest energy in our systems is because we used ethanethiols instead of methanethiols as Barmparis et al. did. This caused a shift of the systems lowest energy to some adjacent angles. The only system that provided its lowest energy at the same configuration with its initial one was $\mathrm{Au}(211)$. The first and the last angles differ by 360 degrees and correspond of course to the same energy due to the periodicity of the potential. We notice that, in the calculation of polynomials we have ensured that the value, as well as their first and second derivatives at the initial and the final angle of calculation, are respectively equal. This was achieved with accuracy between $10^{-10}$ and $10^{-7}$ depending on the examined surface.

3. The second column of each section indicates the dihedral angle translated into the IUPAC/IUB convention, where the angle $\phi$ between the two planes of dihedral angle is zero at cis position $\left(\phi_{\text {rot }}^{(c i s)}=0\right)$.

Moreover, all data have been shifted properly in order to demonstrate the $\phi_{r o t}^{(c i s)}$ at the center of each plot (see Fig. S1). 
Table S1: Data for the dihedral Au-S-C $\mathrm{C}^{(1)}-\mathrm{C}^{(2)}$ fitting for the various surfaces

\begin{tabular}{|c|c|c|c|c|c|c|c|c|c|c|c|}
\hline \multicolumn{3}{|c|}{$\mathrm{Au}(111)$} & \multicolumn{3}{|c|}{$\mathrm{Au}(211)$} & \multicolumn{3}{|c|}{$\mathrm{Au}(221)$} & \multicolumn{3}{|c|}{$\mathrm{Au}(311)$} \\
\hline$(\operatorname{deg})^{a}$ & $(\operatorname{deg})^{b}$ & $(\mathrm{~kJ} / \mathrm{mol})^{c}$ & $(\operatorname{deg})^{a}$ & $(\operatorname{deg})^{b}$ & $(\mathrm{~kJ} / \mathrm{mol})^{c}$ & $(\operatorname{deg})^{a}$ & $(\operatorname{deg})^{b}$ & $(\mathrm{~kJ} / \mathrm{mol})^{c}$ & $(\operatorname{deg})^{a}$ & $(\operatorname{deg})^{b}$ & $(\mathrm{~kJ} / \mathrm{mol})^{c}$ \\
\hline 40 & -176.9 & 0 & -10 & -176.5 & 0.50053 & 10 & -181.1 & 0.22168 & -100 & -177.6 & 0.98058 \\
\hline 50 & -166.9 & 1.92209 & 0 & -166.5 & 0 & 20 & -171.1 & 1.41083 & -90 & -167.6 & 2.0242 \\
\hline 60 & -156.9 & 4.12734 & 10 & -156.5 & 2.9621 & 30 & -161.1 & 2.81249 & -80 & -157.6 & 3.42303 \\
\hline 70 & -146.9 & 7.53486 & 20 & -146.5 & 2.08739 & 40 & -151.1 & 4.06308 & -70 & -147.6 & 4.27523 \\
\hline 80 & -136.9 & 10.84462 & 30 & -136.5 & 5.32198 & 50 & -141.1 & 5.44702 & -60 & -137.6 & 4.54192 \\
\hline 90 & -126.9 & 13.1409 & 40 & -126.5 & 9.18535 & 60 & -131.1 & 4.75483 & -50 & -127.6 & 4.34841 \\
\hline 100 & -116.9 & 14.57073 & 50 & -116.5 & 11.98105 & 70 & -121.1 & 5.02124 & -40 & -117.6 & 3.55137 \\
\hline 110 & -106.9 & 17.17039 & 60 & -106.5 & 15.99277 & 80 & -111.1 & 4.59373 & -30 & -107.6 & 2.24303 \\
\hline 120 & -96.9 & 20.64851 & 70 & -96.5 & 17.38566 & 90 & -101.1 & 4.17872 & -20 & -97.6 & 1.02032 \\
\hline 130 & -86.9 & 22.73943 & 80 & -86.5 & 22.54092 & 100 & -91.1 & 2.9578 & -10 & -87.6 & 0.37624 \\
\hline 140 & -76.9 & 22.88312 & 90 & -76.5 & 20.73711 & 110 & -81.1 & 0 & 0 & -77.6 & 0.1412 \\
\hline 150 & -66.9 & 23.254 & 100 & -66.5 & 17.31129 & 120 & -71.1 & 0.83306 & 10 & -67.6 & 0.99838 \\
\hline 160 & -56.9 & 22.92139 & 110 & -56.5 & 18.7662 & 130 & -61.1 & 1.0445 & 20 & -57.6 & 2.33373 \\
\hline 170 & -46.9 & 22.81019 & 120 & -46.5 & 18.76449 & 140 & -51.1 & 1.50495 & 30 & -47.6 & 5.25309 \\
\hline 180 & -36.9 & 23.06272 & 130 & -36.5 & 20.18011 & 150 & -41.1 & 5.093 & 40 & -37.6 & 7.38145 \\
\hline 190 & -26.9 & 23.22129 & 140 & -26.5 & 19.58138 & 160 & -31.1 & 5.9225 & 50 & -27.6 & 10.69598 \\
\hline 200 & -16.9 & 21.75202 & 150 & -16.5 & 20.05389 & 170 & -21.1 & 9.33662 & 60 & -17.6 & 14.22945 \\
\hline 210 & -6.9 & 21.50898 & 160 & -6.5 & 20.95312 & 180 & -11.1 & 12.62207 & 70 & -7.6 & 16.7203 \\
\hline 220 & 3.1 & 20.04005 & 170 & 3.5 & 18.82854 & 190 & -1.1 & 15.91302 & 80 & 2.4 & 19.02913 \\
\hline 230 & 13.1 & 18.50947 & 180 & 13.5 & 19.6641 & 200 & 8.9 & 15.4219 & 90 & 12.4 & 21.00888 \\
\hline 240 & 23.1 & 16.904 & 190 & 23.5 & 16.377 & 210 & 18.9 & 20.54621 & 100 & 22.4 & 22.58012 \\
\hline 250 & 33.1 & 13.12848 & 200 & 33.5 & 9.82463 & 220 & 28.9 & 17.22396 & 110 & 32.4 & 23.46611 \\
\hline 260 & 43.1 & 10.1456 & 210 & 43.5 & 7.72552 & 230 & 38.9 & 15.69446 & 120 & 42.4 & 24.34837 \\
\hline 270 & 53.1 & 5.85983 & 220 & 53.5 & 4.39806 & 240 & 48.9 & 15.44822 & 130 & 52.4 & 24.77496 \\
\hline 280 & 63.1 & 3.15267 & 230 & 63.5 & 4.733 & 250 & 58.9 & 16.2159 & 140 & 62.4 & 24.10734 \\
\hline 290 & 73.1 & 1.53332 & 240 & 73.5 & 1.15543 & 260 & 68.9 & 20.20456 & 150 & 72.4 & 22.64752 \\
\hline 300 & 83.1 & 0.89677 & 250 & 83.5 & 1.53741 & 270 & 78.9 & 17.38059 & 160 & 82.4 & 21.13333 \\
\hline 310 & 93.1 & 0.56707 & 260 & 93.5 & 2.71241 & 280 & 88.9 & 16.19414 & 170 & 92.4 & 19.28863 \\
\hline 320 & 103.1 & 1.33393 & 270 & 103.5 & 3.21506 & 290 & 98.9 & 17.62774 & 180 & 102.4 & 16.69052 \\
\hline 330 & 113.1 & 1.84689 & 280 & 113.5 & 4.60673 & 300 & 108.9 & 15.50676 & 190 & 112.4 & 12.59822 \\
\hline 340 & 123.1 & 1.83621 & 290 & 123.5 & 5.94061 & 310 & 118.9 & 10.67734 & 200 & 122.4 & 8.59163 \\
\hline 350 & 133.1 & 1.91146 & 300 & 133.5 & 6.52637 & 320 & 128.9 & 6.9842 & 210 & 132.4 & 5.27355 \\
\hline 0 & 143.1 & 2.20951 & 310 & 143.5 & 6.581 & 330 & 138.9 & 5.61106 & 220 & 142.4 & 2.88328 \\
\hline 10 & 153.1 & 1.62349 & 320 & 153.5 & 4.94211 & 340 & 148.9 & 1.8958 & 230 & 152.4 & 1.17682 \\
\hline 20 & 163.1 & 0.66892 & 330 & 163.5 & 3.73802 & 350 & 158.9 & 3.37958 & 240 & 162.4 & 0.17125 \\
\hline 30 & 173.1 & 0.04899 & 340 & 173.5 & 3.21287 & 0 & 168.9 & 0.67759 & 250 & 172.4 & 0 \\
\hline 40 & 183.1 & 0 & 350 & 183.5 & 0.50053 & 10 & 178.9 & 0.22168 & 260 & 182.4 & 0.98058 \\
\hline
\end{tabular}

${ }^{b}$ Angle with respect to $\phi_{\text {rot }}^{(c i s)}=0$, according the IUPAC/IUB convention.

${ }^{c}$ Potential difference bettween the calculated value and the lowest calculated value. 


\section{Fitting plots for calculated potential vs. Au-S-C ${ }^{(1)}-\mathrm{C}^{(2)}$ dihedral angle}

The fitting plots of the four calculated potentials described above are demonstrated in Fig. S1. Fitting data are demonstrated in Table S1. The plots for $\mathrm{Au}(111)$ and $\mathrm{Au}(311)$ fit in the calculated data points quite well, while for the rest of them $(\mathrm{Au}(211)$ and $\mathrm{Au}(221))$ the fitting is not so good at sites near to 0 degrees $\left(\phi_{\text {rot }}^{(c i s)}=0\right.$ ) where the second $\mathrm{C}$ atom seems to "penetrate" into the surface. The reason is that these are non permitted sites because the energy of the system is very high there. However, in spite of the deviation from the expected accuracy, we consider that these potential functions fit quite well the purpose they were constructed for.
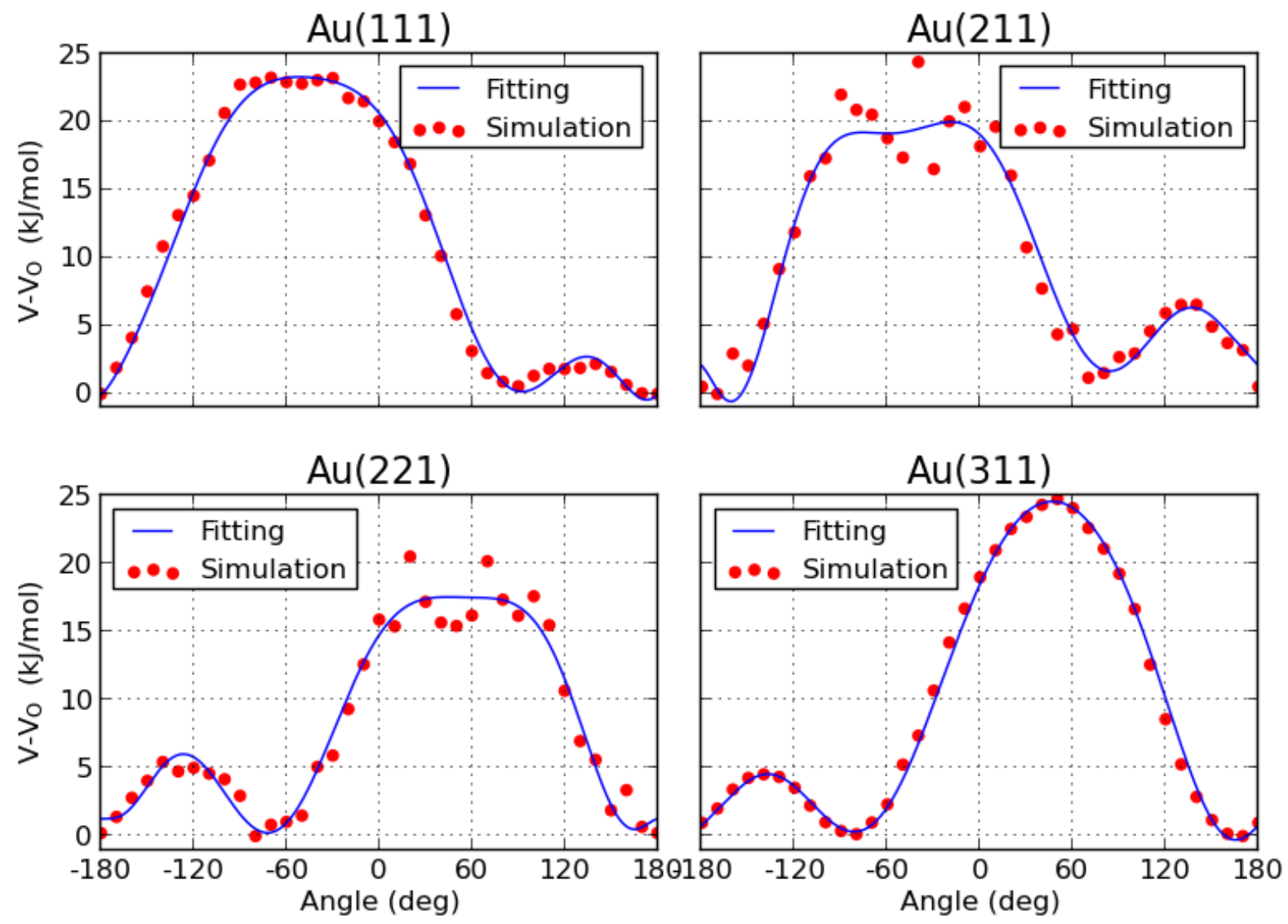

Figure S1: Potential vs. the Au-S-C $\mathrm{C}^{(1)}-\mathrm{C}^{(2)}$ dihedral angle on $\mathrm{Au}(211)$ and (311) surfaces. Red: simulation data, Blue: fitting data. Fitting is not so good in sites where there was strong repulsion to $\mathrm{C}$ atoms from the slab atoms. Angles with respect to $\phi_{\text {rot }}^{(c i s)}=0$, according the IUPAC/IUB convention. 


\section{D structure factor, $S(q)$}

We extracted the structure factor, $S(q)$, for all the examined systems from data of the radial distribution function shown in Fig. 8 of the text. Given the pair correlation function, $g(r)$, in 2D, the structure factor is calculated using the formula

$$
S(q)=1+2 \pi \rho \int g(r) \frac{\sin q r}{q r} r d r
$$

or by its "discretized" form:

$$
S\left(q_{k}\right)=1+2 \pi \rho \sum_{i=0}^{n-1} g\left(r_{i}\right) \frac{\sin q_{k} r_{i}}{q_{k} r_{i}} r_{i} \Delta r
$$

where: $n=900$ is the number of different distances between two chain CMs, $\rho$ is the area density of the CMs, $r_{i}$ is the distance between any two chain CMs, $\Delta r=0.01 \mathrm{~nm}$ is the elementary step of the distance, $g\left(r_{i}\right)$ the value of the radial distribution function for that distance and $q_{k}=\frac{2 \pi}{r_{k}}$.

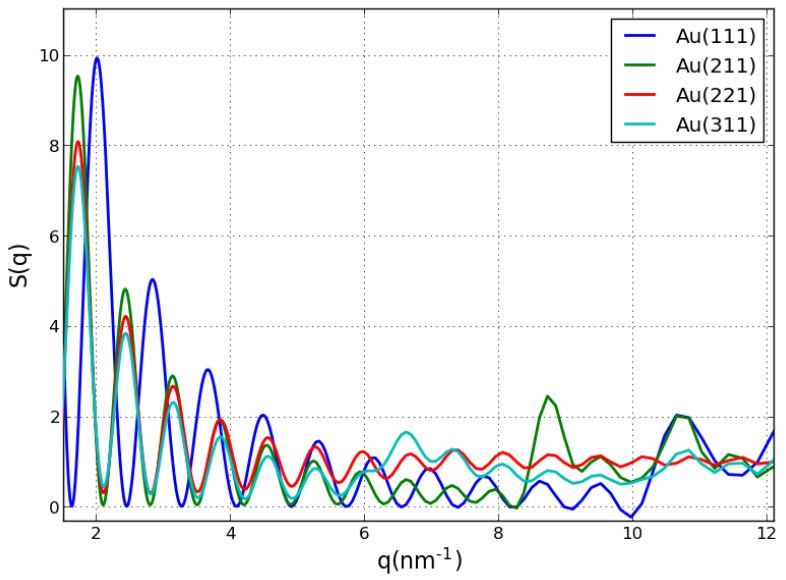

Figure S2: Two-dimensional structure factror for the SAM systems considered in the present study.

The structure factors for the four system are plotted in Figure $S 2$ where we plotted $S(q)$ for $q$ up to $12 \mathrm{~nm}^{-1}$. Comparing this plot with Fig. 8 of the paper, one can observe that there are peaks of $q$ 's $\left(1-12 \mathrm{~nm}^{-1}\right)$ corresponding to distances 0.5 up to $6 \mathrm{~nm}$ between two CMs of the chains indicating the order of both (111) and (211) systems. This is also true for the (311) despite its 
semi-ordered configuration. On the contrary, in the (221) unordered systems there are not such clear peaks which is what we expected because of the system's CMs aperiodicity. 